\title{
Effect of Evidence Based Guidelines on Nurses' Performance to Reduce Complications for Patients after Bone Marrow Transplantation
}

\section{Gailan Abd Elrhman Khalil ${ }^{1}$, Hala Abd El-Salam Sheta ${ }^{2}$ and Rawia Ali Ibrahim ${ }^{3}$}

(1) Bachelor of Nursing,(2009), Menoufia University, Egypt (2) Assistant Professor of Medical Surgical Nursing, Faculty of Nursing, Benha University, Egypt and (3) Lecturer of Medical Surgical Nursing, Faculty of Nursing, Benha University, Egypt

\section{Abstract}

Back ground: Evidence based guidelines after bone marrow transplantation provides nurses with the scientific research to make well-founded decisions and reduce expected complications. Aim of the study: Was to evaluate the effect of evidence based guidelines on nurses' performance to reduce complications for patients after bone marrow transplantation. Research design: Quasi experimental design was utilized. Sample: Convenient sample of all nurses (60) from both sexes who had working in bone marrow transplantation units in Tanta Educational Hospital, and Dar El Salam Cancer Center in Masr Al-Qadimah (Harmel Specialized Hospital previously) and a purposive sample of 30 adult patients from both sexes post-transplant phase were included in the study during nine months. Tools: Three tools were used, I: Structured questionnaire for nurses, II: Nurse's performance observational checklist for BMT patient and III: Patient's assessment sheet. Results: There was positive correlation between knowledge and practice at pre, post and follow up implementation of the guidelines and there was a positive correlation between total nurses' knowledge, practice and patients' early and late complications at post implementation of evidence based guidelines. Conclusion: Implementation of evidence based guidelines has appositive effect in improving nurses' knowledge and practice regarding care of patients undergoing bone marrow transplantation and reducing patients' complications. Recommendations: Evidence based guidelines regarding bone marrow transplantation should be revised periodically and be available in all hospitals in both Arabic and English language.

Key words: Bone marrow transplantation, Evidence based guidelines, Nurses’ Performance, patients' complications.

\section{Introduction}

Bone Marrow Transplantation (BMT) also called Hematopoietic Stem Cell Transplantation (HSCT), it defined as medical procedure used to replace bone marrow cells which damaged by disease or chemotherapy with healthy cells. This procedure involves transplanting blood stem cells, which travel to the bone marrow where they produce new blood cells and promote growth of new marrow (Gulanick \& Myers, 2017). Hematopoietic pro-genitor cells used in transplantation can be obtained from bone marrow, peripheral blood, or placental and umbilical cord blood (Azevedo et al., 2017).
Bone marrow consists of two types; the first type is red bone marrow which produces all red blood cells and platelets in human adults and around 60 to 70 percent of lymphocytes. Other lymphocytes begin life in the red bone marrow and become fully formed in the lymphatic tissues, including the thymus, spleen, and lymph nodes, together with the liver and spleen, red bone marrow also plays a role in getting rid of old red blood cells. The second type is yellow bone marrow which acts as a store for fats. It helps to provide sustenance and maintain the correct environment for the bone to function. However, under particular conditions, such as 
severe blood loss or fever, the yellow marrow may revert to red marrow, yellow marrow tends to be located in the central cavities of long bones, and is generally surrounded by a layer of red marrow with long trabeculae within a sponge-like reticular framework (Clancy and Mcvicar, 2017). There are three types of BMT: allogeneic, autologous, and syngeneic. The cells obtained from someone other than the patient are referred to as allogeneic, whereas cells obtained from the patient are referred to as autologous. In syngeneic BMT, patients receive cells from an identical (monozygotic) twin. The determination of the type of BMT that a patient receives (i.e., allogeneic vs. syngeneic vs. autologous) is based on the disease to be treated, disease state (e.g., initial treatment vs. treatment of recurrent disease), urgency with which the disease needs to be treated, availability of a donor, and time necessary to obtain HSCs from a donor (Bishop and Keating 2019).

Throughout the nursing process, a critical role of BMT nurse is to guide the patient and family through all the information that is needed to understand care options. The emotional context of coping with illness, of grieving the many losses, and of anticipating death can make education and learning a challenge. So this is important for the hospital or the organization to ensure that nursing staff have the knowledge, experience, and training to perform the required tasks. There should be training plan for all staff to maintain a suitable level of competence and ensure that they are only perform the tasks that full within their job description (Gluckman, Niederwieser,\& Aljurf, 2018). Evidencebased guidelines is defined as a conscientious, problem-solving approach to clinical practice that incorporates the best evidence from welldesigned studies, patient values and preferences, and a clinician's expertise in making decisions about a patient's care. Evidence-based guidelines also can be defined as an integration of the best evidence available, nursing expertise, and the values and preferences of the individuals, families and communities who are served (Cornett, 2018).

\section{Significance of the study}

Hematopoietic stem cell transplantation started in Egypt in 1989 on narrow scale , now there are 10 centers of HSCT in Egypt involve 5 centers in Cairo , 2 military centers , 1 center in Alexandria, 1 center in upper Egypt, and 1 center in Tanta city. The number of transplants process is about 580 per year, and the cost about 15,000-20,000 USD (El-Haddad, 2017).

Acute Myeloid Leukemia (AML) accounts for $90 \%$ of all cases of acute leukemia in adults, with an average age at diagnosis of 63 years. In the United States, 18,000 new cases of leukemia are diagnosed each year; 12,000 of which are acute. (Shafik, \&Abd Allah, 2015)

Evidence - based nursing guidelines is the gold standard for quality nursing care delivery. The implementation of evidence based guidelines for patient undergoing BMT is not merely a decisive action for nursing practicing but is also the primary influence on the science of nursing. In addition to, providing patients with complete, comprehensible information essential to confirm the least degree of discomfort throughout the phases of BMT process and minimize the potential complications (ElGazzar, Ahmed, \& El-Tahry, 2019).

\section{Aim of the study}

The aim of the current study was to evaluate effect of evidence based guidelines on nurses' performance to reduce complications for patients after bone marrow transplantation. 


\section{Research hypotheses}

The following research hypotheses were formulated to fulfill the aim of the study:

H1: Nurse's knowledge score regarding care for patient under bone marrow transplantation would be significantly improved after implementing Evidence- based guidelines than their score before.

H2: Nurse's practices score regarding care for patient under bone marrow transplantation would be significantly improved after implementing Evidence- based guidelines than their score before.

H3: Patient's complications after bone marrow transplantation would be reduced.

\section{Subjects and Method}

\section{Research design:}

Quasi- experimental research design was utilized to achieve the study's aim.

\section{Research setting:}

This study was conducted at bone marrow transplantation units in Tanta Educational Hospital, and Dar El Salam Cancer Center in Masr Al-Qadimah (Harmel Specialized Hospital previously).

\section{Sample:}

Nurses: Convenient sample of all nurses (60) who are working in the above mentioned settings from both sexes with different qualifications, their age ranged from 20 to less than 50 years old and willing to participate in the study.

Patients: A purposive sample of 30 adult patients post-transplant phase from both sexes who admitted in the above mentioned settings during nine months were included in this study.

\section{Tools of data collection:}

Three tools for data collection were used as following:
Tool I: Structured questionnaire for nurses: It was designed by the researcher and written in simple clear Arabic language after reviewing recent relevant literatures and scientific references. It was adapted from (Hany et al, 2018). It consisted of two parts to cover the following data:

Part (I): Nurses' demographic characteristics: This part designed to assess nurses' personal characteristics related to their age, gender, marital status, level of education, years of experiences, job description, and previous training about care of BMT patient.

Part (2): Nurses' knowledge regarding care for patient undergoing bone marrow transplantation: It was concerned with assessment nurses' knowledge before and after implementation of evidence - based guidelines.This part was covered the following; 1. General knowledge about the concept of stem cells, bone marrow and their importance, it was consisted of (15) closed end questions. 2- Knowledge about nursing care for a bone marrow transplanted patient, it was consisted of (28) closed end questions. 3Knowledge about possible complications after BMT and how to deal with it was consisted of (12) closed end questions. 4- Knowledge about infection control in BMT units, it was consisted of (15) closed end questions.

\section{Scoring system:}

All knowledge questions were weighted according to the items included two scoring levels for questions were used. Each correct answer was scored (1), each incorrect answer or don't know was scored (zero). The total score for knowledge was (70). Theses scores were summed-up and converted into a percent then categorized as follows: Good $>75 \%$ of the total score, Average $60 \%-75 \%$ of total score and Poor $<60 \%$ of total score . 
Tool II: Nurse's performance observational checklist: It was designed by the researcher after reviewing relevant literature .It was adapted from (Abed El-Hay, 2018). It aimed to assess nurses' practice regarding care of patient undergoing bone marrow transplantation. It included the following: 1- Nursing care of patient pre BMT process (6 items). 2- Caring of patient during BMT process (5 items). 3- Caring of patient after BMT (5 items). 4- Standard precautions in BMT unit (7 items).

\section{Scoring system}

Nurses' performance observational checklist was assigned to score according to number of items and its sub- items. Done practice scored (1), Not done practice scored (0), Total nurses' practice score will be categorized into two levels: Satisfactory $>75 \%$ of total score and Unsatisfactory $<75 \%$ of total score.

Tool (III): Patient's assessment sheet: it was adapted from (Mohamed et al, 2017) it included three parts as following:

Part (1): Patients' demographic characteristics, this part concerned with the assessment of patients' demographic characteristics related to their age, sex occupation, marital status, and level of education.

Part (2): Patients' health history: It was designed by the researcher to assess medical history which includes: nature of disease, years of disease, previous treatments, previous medical intervention and genetic diseases.

Part (3): Patients' sheet for early and late complications after BMT: This tool was adapted from (Kirsch, et al., 2014) to assess early and late complications after BMT process. It included the following parameters: heart rate, oxygenation pattern, heart sounds, blood pressure monitoring, respiratory rate, breathing sounds, temperature, alertness and hemodynamic monitoring, intake and output monitoring, urine and stool culture. Early complications: (low cell count, oral mucositis, breathing problems, infection signs, hemorrhage, numbness and tingling in hands and feet, immunodeficiency, toxicity, neuro psychiatric problems (sleep disturbance, depression and fears). Late complications: (Graft - Versus - Host Disease (GVHD) acute \& chronic, heart problems, kidney problems, liver problems, secondary solid tumors, relapse and death.(

Evidence- based guidelines booklet: It was designed by the researcher through a reviewing of recent related literatures, scientific references as (Elgazzar, Qalawa, \& Eltahry, 2019). This guidelines were planned to cover knowledge and practice related to Evidence- based guidelines for nurses caring patients under bone marrow transplantation, The guidelines content and were included the following items: (introducing the bone marrow, blood components, stem cells, types of bone marrow and their importance, the formation and development of the bone marrow, bone marrow functions, uses of the bone marrow, bone marrow transplant, types of bone marrow transplant, methods for donating bone marrow cells and how to store them, stages of bone marrow transplantation, complications of bone marrow transplantation, patient health education and exit plan, methods of preventing pollution such as: hand washing, personal condoms, intravenous catheterization and care, nursing interventions to reduce complications such as skin care, mouth care, breathing exercises, follow up of kidney functions, and monitor liver function, the appropriate diet for the 
patient, health education for the patient and his family).

\section{Tools validity:}

Once prepared in its preliminary form, the face and content validity were ascertained for comprehensiveness, accuracy, relevance, simplicity, clarity and ambiguity through a jury of five experts from medical surgical nursing department, faculty of nursing, Benha University (two professors and three assistant professors).Also a prepared developed guidelines which covered all items related to nurses caring patients under bone marrow transplantation, based on newest current literature was revised by the same experts and all recommended modifications were done according to their comments and opinions.

\section{Tools reliability:}

Reliability was testing statistically to assure that the tool was reliable before data collection. Internal consistency reliability of the tools was tested using \& Alpha Cronbach Test \&quot; Cronbach`s alpha for knowledge $0.71 \%$, for practice was $0.92 \%$ and for patient complications was $0.83 \%$.

\section{Ethical considerations:}

Written approval was obtained from the research ethical committee of faculty of nursing, also an official permission was obtained from hospital administrators to conduct the study. Each nurse and patient was informed about the purpose, nature and significance of the study, the research assured maintaining anonymity and confidentiality of objective data, every participant has the right to withdraw from the study at any time. Informed consent from the nurses to apply this study was secured. Subjects were assured that this data was not be reused in another research without permission. Patients were assured that their participation is voluntary and they have the right to withdraw from the study at any time without any penalty.

\section{Pilot study:}

pilot study was carried out on $10 \%$ of the studied subject (6 nurses) that was excluded from the total subjects. The pilot study was done in order to test the clarity and applicability of the study tools and the guidelines, to estimate the time required for each tool to be filled in as well as to identify any possible obstacles that may hinder data collection. Based on the results of the pilot study few modifications were done to have more applicable tools for data collection. The pilot study was done one month before starting the study from the end of December 2019 to the end of January2020).

\section{Field work:}

Data collection of the current study was carried out through nine months from the beginning of June 2020 to the end of February 2021. The researcher visited the BMT units at Tanta Educational Hospital, and Dar El Salam Cancer Center in Masr Al-Qadimah (Harmel Specialized Hospital previously) three days' weekly (morning \& afternoon) shifts to collect the data by using previous tools. The study was conducted through the following three phases:

Phase I: assessment phase: The researcher interviewed with available nurses in BMT units and explained the aim of the study and take their approval to participate in the study prior to data collection, then the researcher assessed the nurses' performance level (knowledge and practice) regarding caring of patients with BMT by using questionnaire sheet and observational checklist sheet as following: Firstly, the researcher observed nurses' practice during caring for patients with BMT using the 
observational checklist (Tool II). Based on evidence based guidelines. Each skill was evaluated 3 times and the mean was calculated. The time required to fill each chick list was between 35 - 45 minutes. Secondly, the questionnaire sheets (Tool I) were administered by the researcher to all nurses individually to assess their knowledge about care for BMT patients and explanation of the questionnaire sheet was done by the researcher after an official permission to conduct this study was obtained from the head of the BMT unit and nurses consent. The average time needed for the completion of each interview (by nurses) was between 25 35 minutes and. This period of pretests (knowledge and practice). Based on the finding of the assessment phase, goals priorities and expected out comes were formulated. The researcher planned to provide the nurses with evidence based guidelines in simple Arabic language.

Phase II: Implementation phase: The implementation phase was achieved through educational session' schedule. Each session started by a summary of the previous session, and objectives of the new one. Taking into consideration, the use of Arabic language that suits the nurses' educational level. Motivation and reinforcement during session were used in order to enhance motivation for the sharing in this study. The total numbers of sessions were 12. It divided as follows: eight sessions for knowledge, and four sessions for the practice. The time of knowledge sessions ranged between 45 minutes to 60 minutes. The nurses divided into groups, each group contains (2-3 nurses) to acquire the related information. Each nurse was supplemented with the booklet. The researcher continued to reinforce the gained information, answered any raised questions and gave feedback. The duration of skills sessions ranged between 45 minutes to 60 minutes, and numbers of sessions were 4 sessions for each group (23nurses) in the form of demonstration and redemonstration for each group. Teaching methods were lecture, group discussion, demonstration and re-demonstration. Media utilized were handouts, videos and data show.

Phase III: Evaluation phase: It aimed to evaluate the effect of implementation of Evidence- based guidelines on nursing performance level by compared the results pre, immediate and follow up (after three months) implementation by using the same data collection tools. Also, the researcher evaluates the patient health status for any post-transplant complications (early \& late).

\section{Statistical analysis:}

The collected data were organized, coded, computerized, tabulated and analyzed by using the statistical package for social science (SPSS), version (11). Data analysis was accomplished by the use of number, percentage distribution, mean, standard deviation, and correlation, coefficient; paired t-test was used to test the significance of some variances. A significant level value was considered when $\mathrm{p}<0.05$, and high statistically significant at $\mathrm{p}<0.001$.

\section{Results}

Table (1): Shows that $38.3 \%$ of studied nurses aged between 25 to less than 30 years old with mean age of $29.3 \pm 7.4$ years; females were more prevalent and constituted $70 \%$ of the nurses. Regarding their educational qualification, more than one third $41.7 \%$ had nursing diploma, and $53.3 \%$ have 2 to less than 5 years of experience in the bone marrow transplantation unit. Moreover, it was observed that most of the studied nurses $90 \%$ had not attended training courses.

Figure (1): Illustrates that, only $18.3 \%$ of the studied nurses had good level of 
knowledge at pre implementation of evidence based guidelines, however immediately post implementation more than three quarter $76.7 \%$ of them had good level of knowledge but after three months follow up slightly decline in level of knowledge was observed to $71.7 \%$ respectively.

Figure (2): Reveals that only one thirds $33.3 \%$ of the studied nurses had satisfactory level of practice regarding care of patients under BMT at pre implementation of EBG, while immediately post implementation the majority of them $86.7 \%$ had satisfactory level of practice and slightly decline to $81.7 \%$ at three months of follow up.

Table (2): Shows that more than half $60 \%$ of the studied patients aged between 40 to less than 50 years old and males were more prevalent and constituted more than three quarters $6.7 \%$ of the patients. Regarding marital status, it was found that less than two thirds $63.3 \%$ of them were married. Moreover, it was observed that the majority of studied patients $86.7 \%$ had secondary education level.

Table (3): Reveals that all studied patients $100 \%$ were suffering from chronic disease and more than half $53.3 \%$ of them had chronic disease from two years. Whereas. Most of the patients 90\% had oncology disease. It was noticed that all of the sample $100 \%$ had received chemotherapy As well, had previous bone marrow biopsy.

Table (4): Reveals that $83.3 \%, 100 \%$ and $100 \%$ of studied patients suffer from some early complications as low cell count, breathing problems and infection signs respectively pre implementation of evidence based guidelines. While this complication decreased post implementation of EBG to $10 \%, \quad 26.7 \%$ and $55 \%$ respectively. Regarding late complications this table reveals that $46.7 \%, 66.6 \%, 66.6 \%$ respectively suffer from late complications as GVHD, heart problems and liver problems, while this complications decreased post implementation of EBG $23.3 \%, 63.3 \%$, and $56.7 \%$ respectively, with highly statistically significant differences between pre and post implementation at $\mathrm{p}=0.000$.

Table (5): Reveals that, there was a positive correlation between total knowledge score and total practice score at pre and immediate post implementation of the evidence based guidelines at $\mathrm{P}<0.05$. In addition, there was high significant statistical positive correlation between total knowledge score and total practice score at follow-up implementation of the evidence based guidelines at $\mathrm{P}<0.05$.

Table (6): Shows that, there was no significant correlation between total nurses' practice and patients' early complications at pre implementation of evidence-based guidelines at $\mathrm{p}$ - value $>0.05$. While, there was positive correlation between total nurses' practice and patients' early complications at post implementation of evidence-based guidelines at $\mathrm{P}<0.01$.In addition, there was no significant correlation between total nurses' practice and late complications for patients at pre implementation of evidencebased guidelines at $\mathrm{p}$ - value $>0.05$. While, there was positive correlation between total nurses' practice and late complications of patients $($ GVHD $)=$ chronic, heart problems, kidney problems, liver problems and death) at post implementation of evidence-based guidelines at $\mathrm{P}<0.01$.Also, there was no significant correlation between total patients' knowledge and their early complications at pre implementation of evidence based guidelines at $\mathrm{p}$ - value $>0.05$. While, there was positive correlation between total patients' knowledge and their early complications at post implementation of 


\section{Gailan Abd Elrhman Khalil, Hala Abd El-Salam Sheta and Rawia Ali Ibrahim}

evidence based guidelines at $\mathrm{P}<$ 0.01.Moreover, there was no significant correlation between total patients' knowledge and their late complications at pre implementation of evidence based guidelines at $\mathrm{p}$ - value $>0.05$. While, there was positive correlation between total patients' knowledge and their late complications at post implementation of evidence based guidelines at $\mathrm{P}<0.01$.

Table (1): Distribution of demographic characteristics among studied nurses

\begin{tabular}{|c|c|c|}
\hline \multirow{2}{*}{ Demographic data } & \multicolumn{2}{|c|}{ Total $(\mathbf{n}=60)$} \\
\hline & $\mathbf{N}$ & $\%$ \\
\hline \multicolumn{3}{|l|}{ Age (year) } \\
\hline $20<25$ & 3 & $\overline{5.0}$ \\
\hline $25<30$ & 23 & 38.3 \\
\hline $30<40$ & 16 & 26.7 \\
\hline $40<50$ & 18 & 30.0 \\
\hline \multicolumn{3}{|c|}{ Mean standard } \\
\hline \multicolumn{3}{|l|}{$\overline{\mathrm{Sex}}$} \\
\hline Male & 18 & 30.0 \\
\hline Female & 42 & 70.0 \\
\hline \multicolumn{3}{|l|}{ ducation qualification } \\
\hline Nursing Diploma & 25 & 41.7 \\
\hline Nursing Diploma + Specialization & 6 & 10.0 \\
\hline Technical Nursing Institute & 18 & 30.0 \\
\hline Bachelor of Nursing & $\overline{5}$ & 8.3 \\
\hline Postgraduate studies & 6 & 10.0 \\
\hline \multicolumn{3}{|c|}{ ears of experience in the bone marrow transplantation unit } \\
\hline Less than two years & 3 & $\overline{5.0}$ \\
\hline $2<-5$ years & 32 & 53.3 \\
\hline From $5<-10$ years & 23 & 38.3 \\
\hline More than 10 years & 2 & 3.3 \\
\hline Mean standard & & \\
\hline \multicolumn{3}{|c|}{ Ittended training courses on bone marrow transplantation } \\
\hline Yes & 6 & 10.0 \\
\hline No & 54 & 90.0 \\
\hline \multicolumn{3}{|c|}{ If yes, how many training courses have you attended? ( $N=6)$} \\
\hline One course & 1 & 16.7 \\
\hline Two course & 2 & 33.3 \\
\hline Three course & 1 & 16.7 \\
\hline More than three & 2 & 33.3 \\
\hline
\end{tabular}


Effect of Evidence Based Guidelines on Nurses' Performance to Reduce Complications for Patients after Bone Marrow Transplantation

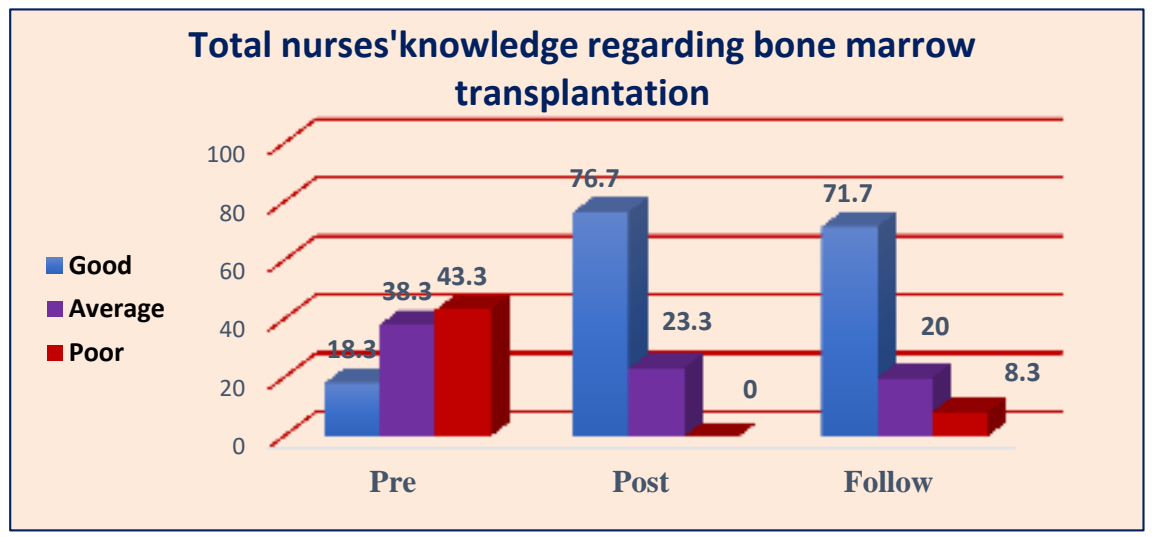

Figure (1): Distribution of total nurses' knowledge regarding bone marrow transplantation

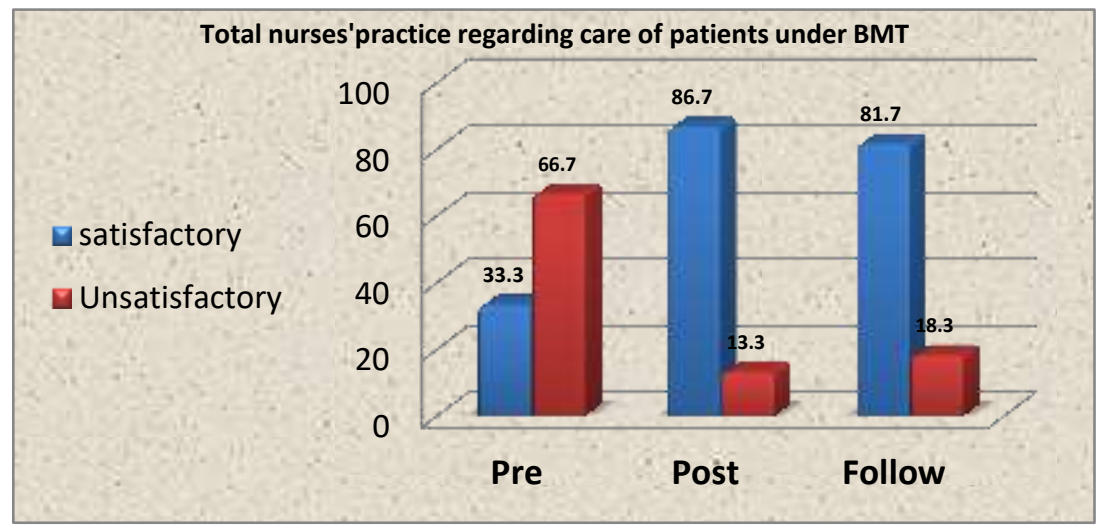

Figure (2): Distribution of total nurses' practices regarding caring of patient under bone marrow transplantation

Table (2): Patients' demographic characteristics $(n=30)$.

\begin{tabular}{|c|c|c|}
\hline \multirow{2}{*}{ Demographic data } & \multicolumn{2}{|c|}{ Total $(n=30)$} \\
\hline & $\mathbf{N}$ & $\%$ \\
\hline \multicolumn{3}{|l|}{ Age (Year) } \\
\hline $20<25$ years & 0 & 0.0 \\
\hline $25<30$ years & 3 & 10.0 \\
\hline $30<40$ & 9 & 30.0 \\
\hline $40<50$ & 18 & 60.0 \\
\hline Mean \pm SD & \multicolumn{2}{|c|}{$40.2 \pm 6.83$} \\
\hline \multicolumn{3}{|l|}{ Sex } \\
\hline Male & 23 & 76.7 \\
\hline Female & 7 & 23.3 \\
\hline \multicolumn{3}{|l|}{ Marital status } \\
\hline Married & 19 & 63.3 \\
\hline Divorced & 11 & 36.7 \\
\hline Widower & 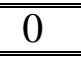 & 0.0 \\
\hline Un married & 0 & 0.0 \\
\hline \multicolumn{3}{|l|}{ Level of education } \\
\hline University education & 3 & 10.0 \\
\hline Secondary education & 26 & 86.7 \\
\hline Illiterate & 1 & 3.3 \\
\hline
\end{tabular}


Table (3): The studied patients according to their medical history $(n=30)$.

\begin{tabular}{|c|c|c|}
\hline \multirow{2}{*}{ Variables } & \multicolumn{2}{|c|}{ Total $(n=30)$} \\
\hline & $\mathbf{N}$ & $\%$ \\
\hline \multicolumn{3}{|l|}{ Suffering from chronic disease } \\
\hline Yes & 30 & 100.0 \\
\hline No & 0 & 0.0 \\
\hline \multicolumn{3}{|l|}{ Years of chronic disease } \\
\hline From one year & 3 & 10.0 \\
\hline From two year & 16 & 53.3 \\
\hline From three years & 3 & 10.0 \\
\hline From more than three years & 8 & 26.7 \\
\hline \multicolumn{3}{|l|}{ Nature of current disease } \\
\hline Hematology disease & 3 & 10.0 \\
\hline Oncology disease & 27 & 90.0 \\
\hline Inherited diseases & 0 & 0.0 \\
\hline Others (specify) & 0 & 0.0 \\
\hline \multicolumn{3}{|l|}{ Previous treatment } \\
\hline Chemotherapy & 30 & 100.0 \\
\hline Radiation therapy & 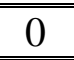 & 0.0 \\
\hline Chemotherapy and radiation & 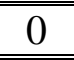 & 0.0 \\
\hline Others (specify) & 0 & 0.0 \\
\hline \multicolumn{3}{|l|}{ Previous medical intervention } \\
\hline BM biopsy & 30 & 100.0 \\
\hline Surgical intervention & 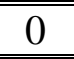 & 0.0 \\
\hline Previous unsuccessful BMT & 0 & 0.0 \\
\hline Others (specify) & 0 & 0.0 \\
\hline
\end{tabular}


Effect of Evidence Based Guidelines on Nurses' Performance to Reduce Complications for Patients after Bone Marrow Transplantation

Table (4): Distribution of the studied patients regarding early and late complications

\begin{tabular}{|c|c|c|c|c|c|c|}
\hline \multirow{2}{*}{\multicolumn{2}{|c|}{ Early Complications }} & \multicolumn{2}{|c|}{ Present } & \multicolumn{2}{|c|}{ Absent } & \multirow{2}{*}{ Pre \& Post } \\
\hline & & $\mathbf{N}$ & $\%$ & $\mathbf{N}$ & $\%$ & \\
\hline \multirow{2}{*}{ Low cell count } & Pre & 25 & 83.3 & 5 & 16.7 & \multirow{2}{*}{$\begin{aligned} \mathbf{X}^{2} & =27.008 \\
P & =0.000\end{aligned}$} \\
\hline & Post & 3 & 10.0 & 27 & 90.0 & \\
\hline \multirow{2}{*}{ Oral mucositis } & Pre & 17 & 56.7 & 13 & 43.3 & \multirow{2}{*}{$\begin{aligned} \mathrm{X}^{2} & =53.49 \\
\mathrm{P} & =0.000\end{aligned}$} \\
\hline & Post & $\overline{11}$ & 38.3 & 19 & 61.7 & \\
\hline \multirow[t]{2}{*}{ Breathing problems } & Pre & 30 & 100 & 0 & 0.0 & \multirow{2}{*}{$\begin{aligned} X^{2} & =67.01 \\
P & =0.000\end{aligned}$} \\
\hline & Post & 8 & 26.7 & 22 & 71.7 & \\
\hline \multirow[t]{2}{*}{ Infection signs } & Pre & 30 & 100 & 0 & 0.0 & \multirow{2}{*}{$\begin{aligned} \mathbf{X}^{2} & =2.707 \\
\mathrm{P} & =0.10\end{aligned}$} \\
\hline & Post & 17 & 55.0 & 13 & 45.0 & \\
\hline \multirow{2}{*}{ Hemorrhage } & Pre & 6 & 20.0 & 24 & 80.0 & \multirow{2}{*}{$\begin{aligned} \mathbf{X}^{2} & =12.31 \\
\mathrm{P} & =0.000\end{aligned}$} \\
\hline & Post & 3 & 10.0 & 27 & 90.0 & \\
\hline \multirow{2}{*}{ Numbness and tingling in hands and feet } & Pre & 12 & 440.0 & 18 & 60.0 & \multirow{2}{*}{$\begin{aligned} \mathbf{X}^{2} & =14.40 \\
\mathrm{P} & =0.000\end{aligned}$} \\
\hline & Post & 3 & 11.7 & 27 & 88.3 & \\
\hline \multirow{2}{*}{ Immunodeficiency } & Pre & 17 & 58.3 & 13 & 41.7 & \multirow{2}{*}{$\begin{aligned} \mathrm{X}^{2} & =7.43 \\
\mathrm{P} & =0.006\end{aligned}$} \\
\hline & Post & 10 & 33.3 & 20 & 66.7 & \\
\hline \multirow{2}{*}{ Toxicity } & Pre & 12 & 40.0 & 18 & 60.0 & \multirow{2}{*}{$\begin{aligned} \mathbf{X}^{2} & =60.00 \\
\mathrm{P} & =0.000\end{aligned}$} \\
\hline & Post & 6 & 20.0 & 24 & 78.3 & \\
\hline \multirow{2}{*}{$\begin{array}{l}\text { Neuro psychiatric problems (sleep disturbance, } \\
\text { depression and fears) }\end{array}$} & Pre & 18 & 26.7 & 12 & 41.7 & \multirow{2}{*}{$\begin{aligned} \mathbf{X}^{2} & =13.807 \\
\mathrm{P} & =0.000\end{aligned}$} \\
\hline & Post & 8 & 26.7 & 22 & 71.7 & \\
\hline \multirow{2}{*}{\multicolumn{2}{|c|}{ Late complications }} & \multicolumn{2}{|c|}{ Present } & \multicolumn{2}{|c|}{ Absent } & \multirow[t]{2}{*}{ Pre \& Post } \\
\hline & & $\overline{\mathrm{N}}$ & $\%$ & $\overline{\mathrm{N}}$ & $\%$ & \\
\hline \multirow{2}{*}{$\begin{array}{l}\text { Graft }- \text { versus }- \text { host disease }(\text { GVHD) }=\text { Acute } \\
\text { Graft }- \text { versus }- \text { host disease }(\text { GVHD })=\text { Chronic }\end{array}$} & Pre & $\overline{14}$ & 46.7 & 16 & 53.3 & \multirow{2}{*}{$\begin{array}{l}\mathbf{X}^{2}=3.59 \\
\mathrm{P}=0.058\end{array}$} \\
\hline & Post & 7 & 23.3 & 23 & 76.7 & \\
\hline & Pre & 20 & 66.7 & 10 & 33.3 & $\mathbf{X}^{2}=0.635$ \\
\hline Heart problems & Post & $\overline{19}$ & 63.3 & 11 & 36.7 & $\mathrm{P}=0.426$ \\
\hline & Pre & 16 & 53.3 & 14 & 46.7 & $\mathbf{X}^{2}=18.261$ \\
\hline Kidney problems & Post & 10 & 33.3 & 20 & 66.7 & $\mathrm{P}=0.000^{* *}$ \\
\hline & Pre & 20 & 66.7 & 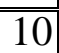 & 33.3 & $\mathrm{X}^{2} 11.380$ \\
\hline Liver problems & Post & 17 & 56.7 & 13 & 43.3 & $\mathrm{P}=0.001$ \\
\hline & Pre & 10 & 33.3 & 20 & 66.7 & $\mathbf{X}^{2}=2.222$ \\
\hline Secondary solid tumors & Post & 7 & 23.3 & 23 & 76.7 & $\mathrm{P}=0.136$ \\
\hline Dolonco & Pre & 12 & 40.0 & 18 & 60.0 & $\mathrm{X}^{2}=1.926$ \\
\hline Relapse & Post & $\overline{5}$ & 16.7 & 25 & 83.3 & $P=0.165$ \\
\hline & Pre & 7 & 23.3 & 23 & 76.7 & $\mathbf{X}^{2}=0.000$ \\
\hline Death & Post & 7 & 23.3 & 23 & 76.7 & $\mathrm{P}=1.000$ \\
\hline
\end{tabular}

Table (5): Correlation between total knowledge and practices scores among studied nurses

\begin{tabular}{|c|c|c|c|c|}
\hline \multicolumn{2}{|c|}{ Variables } & \multicolumn{3}{c|}{ Total knowledge } \\
\cline { 2 - 5 } \multicolumn{2}{|c|}{} & Pre & Immediately post & 3 months follow up \\
\hline Total Practices & $\mathbf{R}$ & 0.297 & 0.307 & 0.332 \\
& $\mathbf{p}$ & $.035^{*}$ & $.017^{*}$ & $.009^{* *}$ \\
\hline
\end{tabular}


Table (6): Correlation between total nurses' practice, knowledge, early and late complications for patients

\begin{tabular}{|c|c|c|c|c|c|}
\hline \multirow{2}{*}{\multicolumn{2}{|c|}{ Complications }} & \multicolumn{2}{|c|}{ Total practice } & \multicolumn{2}{|c|}{ Total knowledge } \\
\hline & & Pre- guidelines & $\begin{array}{l}\text { Post- } \\
\text { guidelines }\end{array}$ & $\begin{array}{l}\text { Pre- } \\
\text { guidelines }\end{array}$ & Post- guideline \\
\hline \multicolumn{6}{|l|}{ Early Complications } \\
\hline Low cell count & $\begin{array}{l}\mathbf{R} \\
\mathbf{p}\end{array}$ & $\begin{array}{l}0.059 \\
.305\end{array}$ & $\begin{array}{l}.164 \\
.033^{*}\end{array}$ & $\begin{array}{l}0.097 \\
.217\end{array}$ & $\begin{array}{l}.179 \\
.036^{*}\end{array}$ \\
\hline Oral mucositis & $\begin{array}{l}\mathbf{R} \\
\mathbf{p}\end{array}$ & $\begin{array}{l}0.096 \\
.215\end{array}$ & $\begin{array}{l}.227 \\
.005 * *\end{array}$ & $\begin{array}{l}0.055 \\
.301\end{array}$ & $\begin{array}{l}.211 \\
.005 * *\end{array}$ \\
\hline Breathing problems & $\begin{array}{l}\mathbf{R} \\
\mathbf{p}\end{array}$ & $\begin{array}{l}0.116 \\
.215\end{array}$ & $\begin{array}{l}.296 \\
.006 * *\end{array}$ & $\begin{array}{l}0.110 \\
.201\end{array}$ & $\begin{array}{l}.214 \\
.005 * *\end{array}$ \\
\hline Infection signs & $\begin{array}{l}\mathbf{R} \\
\mathbf{p}\end{array}$ & $\begin{array}{l}0.091 \\
.229\end{array}$ & $\begin{array}{l}.206 \\
.005 * *\end{array}$ & $\begin{array}{l}0.090 \\
.222\end{array}$ & $\begin{array}{l}.293 \\
.000 * *\end{array}$ \\
\hline Hemorrhage & $\begin{array}{l}\mathbf{R} \\
\mathbf{p}\end{array}$ & $\begin{array}{l}0.099 \\
.275\end{array}$ & $\begin{array}{l}.174 \\
.044 *\end{array}$ & $\begin{array}{l}0.105 \\
.205\end{array}$ & $\begin{array}{l}.155 \\
.047 *\end{array}$ \\
\hline $\begin{array}{l}\text { Numbness and tingling } \\
\text { in hands and feet }\end{array}$ & $\begin{array}{l}\mathbf{R} \\
\mathbf{p}\end{array}$ & $\begin{array}{l}0.093 \\
.235\end{array}$ & $\begin{array}{l}.377 \\
.000 * *\end{array}$ & $\begin{array}{l}0.088 \\
.299\end{array}$ & $\begin{array}{l}.357 \\
.000 * *\end{array}$ \\
\hline Immunodeficiency & $\begin{array}{l}\mathbf{R} \\
\mathbf{p}\end{array}$ & $\begin{array}{l}0.080 \\
.215\end{array}$ & $\begin{array}{l}.192 \\
.031 *\end{array}$ & $\begin{array}{l}0.071 \\
.318\end{array}$ & $\begin{array}{l}.175 \\
0.04 *\end{array}$ \\
\hline Toxicity & $\begin{array}{l}\mathbf{R} \\
\mathbf{p}\end{array}$ & $\begin{array}{l}0.103 \\
.199\end{array}$ & $\begin{array}{l}.299 \\
.006 * *\end{array}$ & $\begin{array}{l}0.111 \\
.200\end{array}$ & $\begin{array}{l}.215 \\
.007 * *\end{array}$ \\
\hline $\begin{array}{l}\text { Neuro psychiatric } \\
\text { problems (sleep } \\
\text { disturbance, } \\
\text { depression and fears) }\end{array}$ & $\begin{array}{l}\mathbf{R} \\
\mathbf{p}\end{array}$ & $\begin{array}{l}0.099 \\
.264\end{array}$ & $\begin{array}{l}.203 \\
.007 * *\end{array}$ & $\begin{array}{l}0.100 \\
.217\end{array}$ & $\begin{array}{l}.205 \\
.009 * *\end{array}$ \\
\hline \multicolumn{6}{|l|}{ Late Complications } \\
\hline $\begin{array}{l}\text { Graft - versus }- \text { host } \\
\text { disease }(\mathrm{GVHD})= \\
\text { Acute } \\
\text { Graft - versus }- \text { host } \\
\text { disease }(\mathrm{GVHD})= \\
\text { Chronic }\end{array}$ & $\begin{array}{l}\mathrm{r} \\
\mathrm{p}\end{array}$ & $\begin{array}{l}0.061 \\
.295\end{array}$ & $\begin{array}{l}.198 \\
.008 * *\end{array}$ & $\begin{array}{l}0.056 \\
.305\end{array}$ & $\begin{array}{l}.167 \\
.007 * *\end{array}$ \\
\hline Heart problems & $\begin{array}{l}\mathrm{r} \\
\mathrm{p}\end{array}$ & $\begin{array}{l}0.099 \\
.286\end{array}$ & $\begin{array}{l}.345 \\
.000 * *\end{array}$ & $\begin{array}{l}0.101 \\
.215\end{array}$ & $\begin{array}{l}.310 \\
.000 * *\end{array}$ \\
\hline Kidney problems & $\begin{array}{l}\mathrm{r} \\
\mathrm{p}\end{array}$ & $\begin{array}{l}0.068 \\
.267\end{array}$ & $\begin{array}{l}.266 \\
.000 * *\end{array}$ & $\begin{array}{l}0.094 \\
.234\end{array}$ & $\begin{array}{l}.264 \\
.000 * *\end{array}$ \\
\hline Liver problems & $\begin{array}{l}\mathrm{r} \\
\mathrm{p}\end{array}$ & $\begin{array}{l}0.103 \\
.291\end{array}$ & $\begin{array}{l}.304 \\
.000 * *\end{array}$ & $\begin{array}{l}0.099 \\
.224\end{array}$ & $\begin{array}{l}.296 \\
.000 * *\end{array}$ \\
\hline $\begin{array}{l}\text { Secondary solid } \\
\text { tumors }\end{array}$ & $\begin{array}{l}\mathrm{r} \\
\mathrm{p}\end{array}$ & $\begin{array}{l}0.050 \\
.333 \\
\end{array}$ & $\begin{array}{l}.168 \\
.041 *\end{array}$ & $\begin{array}{l}0.047 \\
.309\end{array}$ & $\begin{array}{l}.193 \\
.005 * *\end{array}$ \\
\hline Relapse & $\begin{array}{l}\mathrm{R} \\
\mathrm{p}\end{array}$ & $\begin{array}{l}0.080 \\
.274\end{array}$ & $\begin{array}{l}.192 \\
.011^{*}\end{array}$ & $\begin{array}{l}0.091 \\
.300\end{array}$ & $\begin{array}{l}.144 \\
.011^{*}\end{array}$ \\
\hline Death & $\begin{array}{l}\mathrm{R} \\
\mathrm{p}\end{array}$ & $\begin{array}{l}0.099 \\
.208\end{array}$ & $\begin{array}{l}.188 \\
.015^{*}\end{array}$ & $\begin{array}{l}0.067 \\
.200\end{array}$ & $\begin{array}{l}.170 \\
0.01 *\end{array}$ \\
\hline
\end{tabular}




\section{Discussion}

Nurses in BMT units play a key role in management and prevent the early and late complications of BMT process and require specific training and using Evidence based guidelines provides nurses with the scientific research to make well-founded decisions and reduce expected complications (Kenyon,M. , \& Babic,A.2018). The current study aimed to evaluation the effect of evidence based guidelines on nurses' performance to reduce complications for patients after bone marrow transplantation.

Distribution of demographic
characteristics of studied nurses:
Regarding to age; the results of the present study reported that more than one third of the studied nurses aged between 25 to less than 30 years old. It may be due to the unit was recently established and the young age nurses able to perform tasks in the unit effectively. This result was in the same line with Mohamed and EL-Sayed, (2015) who reported in their study about "Nurses' knowledge regarding stem cells therapy" that more than half of nurses were aged from 2025 years. Also, this result agreed with AbdElrahaman, (2011), who found in his study about "Evaluating the knowledge and practice of nurses toward infection control measures during bone marrow aspiration" that more than two thirds of nurses were in age group $20<30$ years. While this study result was contradicted with the study of Mahdy,(2009) documented in his study about "the effect of training program on staff nurses performance toward caring of patients undergoing organs and tissue transplantation" that the majority of studied nurses age ranged between 22-24 years.

As for gender, the results of the present study revealed that more than two third of studied nurses were females. It may be due to over all relation of male nurses to female nurses were less in nursing profession. This result was consistent with Wright, (2013) who report in his study entitled "Intensive care nurses' knowledge and practices regarding infection control standard precautions at a selected Egyptian Cancer Hospital" that most critical care nurses were females. Also, this finding was in line with Karaly, \& Abo El-Fetoh, (2019) who reported in their study about "Effect of an educational guideline on nurses' performance caring for patients post liver transplantation" stated that, the majority of the study nurses were females. While the findings of the current study was contradicted with the study of Taha , (2014) who studied "Comparative study between two nutritional regimes on wound healing of moderate burned patient. Unpublished doctoral dissertation," and reported that more than half of them were males.

In relation to educational qualification, the result of the current study showed that, that more than one third had nursing diploma, this could be explained in light of the known fact that nursing job in Egypt was exclusive on female only till few years ago and number of nurses graduated from diploma was higher than Bachelor. This result was congruent with Mohamed and Sayed, (2015) who reported the majority of nurses who are working in BMT unit had diploma degree in nursing. These findings are supported by that study conducted by Woolery, et al., (2012), whose study was about "Ambulatory care of the bone marrow transplant client" and reported that the highest percentage of the oncology nurses had nursing diplomas. But this result was contradicted by Ali, et al.,(2019) in his study entitled "Effect of Teaching Program on Nurse's Performance Regarding Bone 
Marrow Transplantation" reported that majority of nurses were bachelor degree.

Regarding to years of experience; the findings of the present study clarified that more than half of studied nurses had two to less than five years of experience in the bone marrow transplantation unit, this might be related to the modernity of the BMT medical field in Egypt and these BMT centers are newly established medical centers. This result was on the same line with Ali, et al., (2019) reported that more than half of the studied nurses had years of experience from one to less than five years. But Khalil, (2013) disagree with this results and reported in his study entitled "Impact of implementing a designed nursing intervention protocol on nurses' knowledge and practice regarding patients undergoing blood transfusion" that less than two-third of nurses had an experience of more than 10 years .

\section{Regarding to attending training} courses on bone marrow transplantation; the finding of the current study revealed that majority of the studied nurses had not attended training courses on bone marrow transplantation. it may be due to the shortage number of nurses in BMT unit and they have over load in the work that prevent them from attendance any training courses . This findings were correspondent to Shaban, (2018) who studied "Effect of educational intervention on nursing students' performance regarding umbilical cord blood (UCB) collection for stem cells" and found that the majority of the studied subjects' didn't have any previous information. Also, this finding was in agreement with Amin, et al., (2016) who found in their study entitled "Knowledge regarding umbilical cord stem cell therapy among staff nurses with the view to develop self-instructional module" that most of the studied nurses had not attended training course regarding umbilical cord stem cell therapy.

Distribution of total nurses'
knowledge regarding bone marrow
transplantation, The results of current revealed that less than one fifth of nurses had good level of knowledge pre implementation of evidence based guidelines and improved after implementation of guidelines to three quarter of studied nurses but after three months follow up, there was a slight decline in nurses total knowledge. According to the researcher point of views that, the low level of nurse's knowledge pre implementation of evidence based guidelines may be related to absent of continuous training and courses, while there was an improvement of nurse's knowledge post implementation of EBG which indicated the effectiveness of EBG implementation and the quick responses of nurses. This result supported by Ali, et al., (2019) who documented that there was significant improvement regarding total levels of nurses' knowledge about bone marrow transplantation before and after implementation of teaching program. Also, this result agreed with Karaly, \& Abo ElFetoh, (2019) who stated that all of the study nurses had an unsatisfactory score regarding their total knowledge at preeducational guidelines implementation phase, which may be due to unavailability of posters and booklets and training courses while the satisfactory score improved post-educational guidelines implementation, while this improvement lowered slightly in the followup phase. This might be due to retention of knowledge with the time factor. This finding was in consistent with Sakr, et al. , (2019), who reported in their study about "Effect of intervention guidelines on nursing performance regarding patients with angina " that the unsatisfactory level of nurses' knowledge before implementing nursing 
intervention guidelines, this deficiency in knowledge is owing to one or more of the following reasons, lack of orientation program before work; lack care conferences during work; non availability of procedure books; especially prepared for the critical care areas; and lack of direction and nurses' appraisal about patient care. Also, this results was in agreement with Abed El-Hay, (2018 ), who reported in his study that there were highly significant improvements among nurses' knowledge regarding; source, causes, types, risks, complications and management of patients' pre, during and after bone marrow transplantation throughout all intervention periods of the study, also there were significant improvement regarding total levels of nurses' knowledge about management of patients undergoing bone marrow transplantation throughout all intervention periods of the study. The results were similar also to findings of a study conducted by Shafik, \&Abd Allah, (2015) who reported in their study about "Improving the Quality of Nursing Care for Patients with Leukemia in Day Care Units Through Nursing Education " that there was an improvement after program implementation and these improvements revealed a statistically significant difference in total nurses' knowledge pre - post nursing education program. Also, this results was supported by Lovis, (2010) who found in his study entitled " Evaluate the effectiveness of selfinstructional module on knowledge of placental stem cell and its utilization" that the majority of staff nurses have poor knowledge in the pre-test and most of the staff nurses had good knowledge regarding cord blood collection and stem cells and its utilization.

Total nurses' practices regarding caring of patient under bone marrow transplantation The results of current study revealed that only one thirds of the studied nurses had satisfactory level of practice regarding care of patients under BMT at pre implementation of EBG, while immediately post implementation the majority of them had satisfactory level of practice and slightly decline at three months of follow up. This might be due to the effectiveness of evidence based guidelines implementation in enhancing nurse's practices through BMT phases. These results were in accordance with Ali, et al, (2019) who revealed that all of nurses had unsatisfactory practices regarding care of patients with bone marrow transplantation before teaching program intervention, while all of them had satisfactory practices after teaching program intervention. Also, these results were in the same line with Abed El-Hay, (2018) mentioned that there were highly significant improvements in the nurses' practice regarding management of patients' pre and during bone marrow transplantation. In addition to Sherif, (2016) reported that there was significant improvement in nurses' practice level post program implementation.

Patients'

demographic characteristics regarding to gender the current study revealed that more than half of the studied patients aged between 40 to less than 50 years old. These results agreed with Elgazzar, et al.,(2019) whose study was "The efficiency of evidence-based guidelines on performance, perception, and satisfaction of patients undergoing bone marrow biopsy " reported that less than half of the studied patients were in the age group of 4150years.Regarding marital status, it was found that less than two thirds of them were married. These results agreed with Elgazzar, et al.,(2019) who reported that more than half of the studied patients were married. Regarding to sex, the current study revealed that males were more prevalent and 
constituted more than three quarters of the patients. This finding is supported by Bajaj, et al., (2013) who documented in his study entitled "quality of life after chemotherapy medications" that males are more affected with cancer than females. Also Abd Elhameed, (2011), found in his study entitled "evaluation the effect of designed implemented nurse's educational program on minimizing incidence of complications for patients with colostomy" that more than half of study subjects were males. But this result was in contrast with Mahdy, (2009) who found that females are more affected than males. Regarding the education level, it was observed that the majority of studied patients had secondary education level.

Regarding to the studied patients according to their medical history, the current study revealed that all studied patients were suffering from chronic disease. Whereas, most of the patients had oncology diseases. It was noticed that all of the sample had received chemotherapy, as well, had previous bone marrow biopsy. These results revealed the indication of BMT which depends on numerous factors such as the disease type, stage and response to previous treatment. BMT is a life- saving treatment for many diseases such as aplastic anemia, leukemia, inherited blood diseases, sickle cell disease, and autoimmune diseases. These results were in the same line with Mahdy, (2009), who found that about two thirds of the studied patients had suffered from leukemia due to the cause of BMD. Also, Mohamed, (2007) mentioned in his study entitled "bone marrow transplantation coordinator" that the most common causes of BMT are the auto immune disorders. In addition to Sherif , (2016) who found that more than one third of studied patients had cancer diseases and more than one quadrant of them had blood diseases.
Regarding early and late complications among studied patients regarding to early complications; the current study finding showed that most of studied patients suffered from some early complications as low cell count, breathing problems and infection signs pre implementation of evidence based guidelines. While this complication decreased post implementation of EBG to less than half. This results in accordance with National marrow donor program, (2017) which stated that in its annual report about "A centralized cord blood registry to facilitate allogeneic, unrelated donor umbilical cord blood transplantation" that after having bone marrow transplantation the risk of developing an rejection will increase this is because the conditioning regimen receive before the transplant will weaken the immune system and the risk for infection. This results in agreement with Cutler, et al., (2005) in his study about "mucositis after allogeneic hematopoietic stem cell transplantation" who found that oral mucositis occurs in up to three quarter of recipients of high-dose chemo radiotherapy conditioning regimens used for allogeneic hematopoietic stem cell transplantation (HSCT). Also, Nichols, (2017), in his study about "management of infectious complications in the hematopoietic stem cell transplant recipient" reported that the risk for infectious complications after HSCT is dependent on multiple factors, which include underlying disease status, patient age, type of transplant, conditioning regimen, and history of previous infections (including pre transplant viral serostatus).This result is supported also by Mersal, (2011) who noticed "after training programs for patients undergoing BMT" that the risk of rejection may be diminished and withdrawal gradually. 
Regarding to late complications; This result revealed that about two third suffered from late complications as GVHD, heart problems and liver problems, while this complications decreased post implementation of EBG to less than half of the studied patients, with highly statistically significant differences between pre and post implementation. This results in the same line with Abd El- Moniem, et al., (2008) who reported in his study about "management of complications followed bone marrow transplantation "that most of patients after BMT were complained of some cardiac diseases pre implementation of educational program. Also Singh, (2015), stated in "the researcher process in nursing $\left(5^{\text {th }}\right.$ ed)" that promoting nurse's knowledge will decrease complications of their patients and he suggested that increasing patient's education regarding disease and treatment modalities might enhance compliance. but this result disagree with Mahdy, (2009), who noticed that renal complications were increased after kidney and BMT transplantation related to immunosuppressant medications. Also, the current study revealed that there were significant improvement in the studied patient's complications post implementation of EBG, this may be due to the increasing in nurses' level of knowledge and practice regarding caring of BMT after implementation of EBG.

\section{Regarding to correlation between} total knowledge and total practices scores among studied nurses; the results of current study revealed that there was a positive correlation between total knowledge score and total practice score at pre and immediate post implementation of the evidence based guidelines. In addition, there was high significant statistical positive correlation between total knowledge score and total practice score at follow-up implementation of the evidence based guidelines. This might be due to the evidence based guidelines improved level of nurses' knowledge which affects positively their practice regarding caring for patients undergoing bone marrow transplantation. This result was in the same line with the study done by Karaly \& Abo Elfetoh, (2019) reported that there was statistically significant correlation between total knowledge and practice of study sample. Also, this result supported by Ali, et al., (2019) documented that there was a positive correlation between total knowledge score and total practice score pre and post teaching program intervention. Also Debock, et al., (2014), reported in their study about principles and practice of supportive oncology nursing that higher scores in nurses' knowledge were related to higher practice. This result confirmed by Jefford and Tattersall, (2010), who clarified in their study about bone marrow transplantation ( oncology nursing secrets) that the nurse need for promoting their knowledge and level about BMT was positively related to their need for enhancement their practice. As well as Bayoumi , Kamel , \&Fathalla, (2017), revealed in their study about "Guideline Education Program about Nursing Ethics in Pediatric Oncology Unit", that there was a positive correlation between nurses' knowledge and practice regarding nursing ethics toward children in pediatric oncology unit at post program intervention. Also Shafik, \&Abd Allah, (2015), reported that there was a statically significant correlation between total nurses' knowledge and total nursing performance score pre \& post program implementation. In addition to this findings was conducted by Hosseini et al., (2015) who conducted a study on "critical care nurses' knowledge, attitude, and practice toward their role in the organ donation process from brain-dead patients 
and factors influencing" mentioned that there were significant direct correlations between knowledge and practice of the study nurses. On contrary, Gijare, (2012), said in his study entitled "Effectiveness of teaching on infection control practices among health care professionals" that there was no correlation between knowledge and practice.

As regards correlation between total nurses' practice, knowledge, early and late complications for patients at pre and post implementation of evidence-based guidelines; the current study results revealed that there was no significant correlation between total nurses' knowledge and patients' early and late complications at pre implementation of evidence based guidelines .While, there was positive correlation between total nurses' knowledge and patients' early and late complications at post implementation of evidence based guidelines .Also, there was no significant correlation between total nurses' practice and patients' early and late complications at pre implementation of evidence-based guidelines .While; there was positive correlation between total nurses' practice and patients' early and late complications (GVHD), chronic, heart problems, kidney problems, liver problems and death) at post implementation of evidence-based. These results in the same line with Sherif, (2016), who revealed that there was significant improvement in studied patient's complications after implementation of educational program. Also Acharya, (2019) reported in their study about "knowledge and practice on prevention of complications of diabetes mellitus among patients with diabetes in a tertiary hospital" that there was an average positive relationship between knowledge and practice on prevention of diabetes complications.

\section{Conclusion}

There were statistical significant improvements of nurses' knowledge and practice regarding care for patient under bone marrow transplantation post implementation of evidence based guidelines than pre intervention period. Also, it was noticed that, there were statistical significant positive correlation between total knowledge and total practice at pre, post and follow up implementation of evidence based guidelines. Clearly, there was positive correlation between total nurses' knowledge and early and late complications of patient at post implementation of evidence based guidelines. Also, it was observed a positive correlation between total nurses' practice and early and late complications of patients at post implementation of evidence based guidelines which supported the study hypotheses.

\section{Recommendations}

- The need for continued nursing education and in service training programs about bone marrow and stem cell transplantation should be regularly organized by hospital administration for staff nurses in order to equip them with adequate knowledge and practice related to care of patients.

- In addition, Evidence based guidelines regarding bone marrow transplantation should be revised periodically and be available in all hospitals in both Arabic and English language.

- Evidence based guidelines regarding bone marrow and stem cell transplantation should be incorporated in the different nursing curricula to strengthen graduates' level of understanding and practice.

- Moreover, further researches with large sample from nurses and student nurse are recommended to update information among them.

-The educational intervention and patient's psychosocial support should be included in 
routine nursing care as a protocol before and after bone marrow and stem cell transplantation surgery and at the time of follow up phase.

\section{References}

Abd El Moniem., (2008). The new egyption journal of medicine, management of complications followed bone marrow transplantation, Vol, 29., No7. pp60-62.

Abd- Elrahaman, E., (2011). Evaluation the knowledge and practice of nurses toward infection control measures during bone marrow aspiration at Alexandria university hospitals, Doctorate Thesis, faculty of nursing, Alexandria university, p 123

Abd- Elhamed, (2011). Evaluating the effect of designed implemented nurse's educational program in minimizing incidence of complications for patients with colostomy. Doctorate Thesis, Medical- Surgical Nursing, Faculty of nursing, Alexandria university, p127.

Abed El-Hay, S., (2018). Effect of Educational Guidelines on Nurses` Performance Regarding management of Patients undergoing Bone marrow Transplantation.”.” IOSR Journal of Nursing and Health Science (IOSR-JNHS), vol. 7, no. 1 , pp. $69-80$

Acharya, S. (2019). Perception of Dental Scientists and Post-Graduate Students Regarding Future Prospects of Stem Cells in Dentistry. Acta stomatology Croat, 47(4), 312-21.

Ali, A, Mohamed,M., Sayed,D. \& Ahmed,N.( 2019). Effect of Teaching Program on Nurs's Performance Regarding Bone Marrow Transplantation. Assiut Scientific Nursing Journal, Vol , (7) No, (18).pp12-21.

Amini, F., Lai1 ,D., \& Ramasamy, S. , 2016. knowledge, Awareness and Perception of Stem Cells Research amongst Malaysian
Medical Students, Regenerative Research. 2016; 4(2): 25-30.

Azevedo, I., Cassiano, A., Carvalho, J., et al., (2017). Nursing Care for Hematopoietic stem cell transplant recipient and their families. Article review, Doi: 10.15253/2176783-2017000400019. available at: www.revistarene-ufc-br

Bajaj, L., et al., ( 2013). quality of life after chemotherapy medications; the impact of cancer care, journal of nursing care and research, 27(6). ; pp 864-868

Bayoumi, H., \& Mahmoud, F., (2017). Effect of education program on nurses' knowledge and practice regarding care of central venous line in pediatric hemodialysis: evidence-based practice guidelines. Egyptian Nursing Journal, 14(2), 87

Bishop, M., \& Keating, A., (2019). hematopoietic stem cell transplantation, Goldman- cecil Medicine, 26th edition, Elsevier, available at; https://www.clinicalkey.com

Clancy, J., \& Mcvicar, A., (2017). Physiology and Anatomy for nurses and health care practitioners, a homeostatic approach, 3rd Edition, HODDER ARNOLD and Hachette company. Available at; https://www.taylorandfrancis.com

Cornett, J., (2018). What are the benfits of evidance based practice in nursing, the Nest, availlable at; //www.women.theenest.com accessed at $12 \mathrm{am}$.

Cutler, C., Kim ,T., Sun, L., et al. (2005). Donor-specific anti-HLA antibodies predict outcome in double umbilical cord blood transplantation. Blood. 2011;118(25):66916697. [PMC free article] [PubMed] [Google Scholar]

DeBock, A., Berger, A., Portenoy, R., etal., (2014). Principles and practice of supportive oncology nursing. 6th edition, Philadelphia; Lippincott, Williams and wilkins, pp 55-65. 
Elgazzar,S.,

Qalawa,S. \&

Eltahry,S.(2019).The Efficiency of

Evidence-Based Guidelines on Performance, Perception, and Satisfaction of Patients Undergoing Bone Marrow Biopsy, International Journal of Nursing, Vol. 6, No. 1, pp. 72-82

Elhaddad,A. (2017). HSCT in Egypt an over view-WBMT. Available at: https://www.wbmt.org

Gijare, (2012), Effectiveness of teaching on infection control practices among health care professionals Corpus ID: 55909682. available at

https://www.semanticscholar.org /pape

Gluckman,E. ， Niederwieser, D., \&Aljurf,M. (2018):Establishing a Hematopoietic stem cell transplantation unite: A practical Guide,Springer AG, P222Available at: https://doi-org /10.1007/978-3-319-59358-6

Gulanick, M., \& Myers, J.(2017). Nursing Care Plans: Diagnosis, intervention, and outcomes, 9th Edition, Elsevier comp, USA, ISBN: 978-0-323-42818-7

Hany,S. , Ahmed,R. ,\& Sharshor,S. (2018): Effect of Educational Guidelines on Nurse's performance regarding management of patient undergoing bone marrow transplantation, IOSR Journal of Nursing and Health science, e-ISSN:2320-1959. Volume 7, Issue 1, PP69-80

Hosseini S, Manzari Z, Khaleghi I (2015). ICU nurses' knowledge, attitude, and practice towards their role in the organ donation process from brain-dead patients and factors influencing it in Iran. Int J Organ Transplant Med 6: 105-113.

Jefford \&Tattersall, (2010). Oncology nursing secrets; bone marrow transplantation,3rd edition. Mosby, Philadelphia , p 236
Karaly, S., \& , Abo Elfetoh, E., (2019). Effect of an educational guideline on nurses' performance caring for patients post liver transplantation, Volume: $16 \quad$ Issue : $3 \quad$ Page: 115-127. DOI: 10.4103/ENJ.ENJ_8_19.

Kenyon,M. ， \& Babic,A.(2018): The European Blood and Marrow transplantation ,Text Book for Nurses, Under the Auspices of EBMT, Springer, ISBN: 978-3319.50025-6, Available at: https://doi-org /10.1007/978-3-319-50026-3

Khalil, S., (2013). Impact of implementing a designed nursing intervention protocol on nurses' knowledge and practice regarding patients undergoing blood transfusion. Med J 81:163-171.

Khalil, A. M. \& Sharshor S. (, 2016). Pediatric Nurses Knowledge, Awareness and Attitude towards Application of Stem Cells Therapy in Children, IOSR Journal of Nursing and Health Science, 5( 4): 88-96.

Kirsch M., et al, (2014)., Nurses' Practice Patterns in Relation to Adherence-Enhancing Interventions in Stem Cell Transplant Care: A survey from the Nurses Group of the European Group for Blood and Marrow Transplantation, European journal of cancer care, 23: 607-615.

Lovis, V., (2010). A study to evaluate the effectiveness of self-instructional module on knowledge of placental stem cell and its utilization among staff nurses in selected hospitals at Mangalore. Published M.Sc. Nursing thesis submitted to Alva's College of Nursing Moodbidri, Rajiv Gandhi University of Health Sciences Karnataka, Bangalore; 60. Accessed on: 5 January 2020.

Mahdy, A., (2009). effect of training program on staff nurses performance and empowerment toward care of patient undergoing organs and tissue transplantation , Doctorate Thesis, faculty of nursing, Benha University. 
Mersal, S., (2011). assessment of knowledge and attitude of nurses regarding care of their patients under going chemotherapy, thesis MD, Mansoura university, faculty of nursing, Medical Srgical Department, p.110

Mohamed, (2007). Bone marrow transplantation coordinator, Vol92- 20/ April/post graduate medicine., pp12-15.

Mohammed , S., \& EL Sayed H., (2015). A. Knowledge and Attitude of Maternity Nurses Regarding Cord Blood Collection and Stem Cells: An Educational Intervention. Journal of Nursing Education and Practice, 5(4): 58-69. Mohamed, S. -, Salem, G., Mohamed, H., (2017). Effect of Selfcare Management Program on Self-efficacy among Patients with Colostomy American Journal of Nursing Research. 2017, 5(5), 191-199. DOI: 10.12691/ajnr-5-5-5. Published online: October 28, 2017 Volume 5, $2017 \quad$ - Issue $5 . \quad$ available at: https://www.sciepub .com/journal /ajnr

National Marrow Donor Program (2017). HLA matching: finding the best donor or cord blood unit. available at; http://marrow.org

/Patient/Transplant_Process/Search_Process/ HLA_Matching_Finding_the Best Donor or Cord Blood Unit. aspx. Accessed November 14.

Nichols ,G.,( 2003). Management of infectious complications in the hematopoietic stem cell transplant recipient. J Intensive Care Med. 2003;18:295-312. DOI: 10.1177/0885066603258009. Available at: https://www.medicalnewstoday.com.

Sakr, M., Metwaly, E., \& Taha, N., (2019). Effect of intervention guidelines on using performance regarding patients with angina. Medical Surgical Nursing Department, Faculty of Nursing, Zagazig University, Zagazig, Egypt. Year: 2019 Volume : 16 Issue : 2 Page : 7079. DOI: 10.4103/ENJ.ENJ_4_19.

Shaban, M., (2018). Effect of Educational Intervention on Nursing Students'
Performance Regarding Umbilical Cord Blood Collection for Stem Cells. IOSR Journal of Nursing and Health Science (IOSR-JNHS), 7(6), 68-81.

Shafik, S., \& Abd Allah , E., (2015). Improving the Quality of Nursing Care for Patients with Leukemia in Day Care Units Through Nursing Education, . American Journal of Nursing Science. Vol. 4, No. 3, 2015, pp. 63-72. doi: 10.11648/j.ajns.20150403.14.

Sherief , H., (2016). Assessment of nurses' performance in caring for patients with bone marrow transplantation [unpublished, master thesis] Benha, Egypt: Faculty of Nursing, Benha University.

Singh, M., (2015). The research process in nursing congress, 5th edition, Black Well. Pp 537-540

Woorlery, A., Buchsel, P \& parchem, C., (2012): Ambulatory care of the bone marrow transplant client .A multicentre case-control study .J Epidermal Community Health, Jun: 54(6): 421-426.

Wright, J., (2013). The miracle of kathleen: the kathleen wright story. New York: Jerry Wright Company. 48. 
تاثير الارشادات القائمة علي الادلة علي اداء التمريض للحد من المضاعفات للمرضي بعد زرع

\section{النخاع العظمي}

جيلان عبدالرحمن خليل - هاله عبدالسلام شتا- راويه علي إبر اهيم

زرع نخاع العظم أو زرع الخلايا الجذعية المكونة يُعرَّف بأنه إجر اء طبي يستخدم لاستبدال خلايا نخاع

العظم التي تضررت بسبب المرض أو العلاج الكيميائي بالخلايا السليمة. وإنه خيار علاجي قوي لمجموعة من أمر اض الأور ام الدموية والأور ام المناعية الموروثة أو المكتسبة ور اثيًا.لذا هدفت هذه الدراسة الي تقييم تاثير الارشادات القائمة علي الادلة علي اداء التمريض للحد من المضاعفات للمرضي بعد زرع النخاع العظمي وقد أجريت هذه الدر اسة في وحدات زرع النخاع العظمي في كل من مستشفى طنطا التعليمي ومستشفي دار السلام بمصر القديمة علي عدد من الممرضات العاملات في هذه الوحدات و عددهم . ؟ ممرضه و . م مريض. حيث كثفت النتائج عن أن هناك فروق ذات دلالات إحصائية بين مستوي معلومات وممارسات الممرضات ومؤهلاتهم وسنوات الخبرة قبل وبعد تنفيذ الإرشادات التعليمية. كانت هنالك علاقة إيجابية ذات دلالة إحصائية بين مستوي معلومات وممارسات الممرضات تجاه رعاية مرضي زرع النخاع العظمي قبل وبعد تنفيذ الإرشادات التعليمية. وأوصت الدراسة بأن هناك حاجة إلي تنفيذ برامج توجيهية للممرضات العاملات في وحدات زرع النخاع العظمي لتحديث مستوي معلوماتهم وتحسين ممارساتهم تجاه رعاية المرضي الخاضعين لعملية زر ع النخاع العظمي . 